PROCEEDINGS OF THE

AMERICAN MATHEMATICAL SOCIETY

Volume 138, Number 7, July 2010, Pages 2335-2339

S 0002-9939(10)10288-3

Article electronically published on February 4, 2010

\title{
THE WEAK LEFSCHETZ PROPERTY AND POWERS OF LINEAR FORMS IN $\mathbb{K}[x, y, z]$
}

\author{
HAL SCHENCK AND ALEXANDRA SECELEANU
}

(Communicated by Bernd Ulrich)

\begin{abstract}
We show that an Artinian quotient of an ideal $I \subseteq \mathbb{K}[x, y, z]$ generated by powers of linear forms has the Weak Lefschetz Property. If the syzygy bundle of $I$ is semistable, the property follows from results of Brenner-Kaid. Our proof works without this hypothesis, which typically does not hold.
\end{abstract}

\section{INTRODUCTION}

Let $I \subseteq S=\mathbb{K}\left[x_{1}, \ldots, x_{r}\right]$ be an ideal such that $A=S / I$ is Artinian. Then $A$ has the Weak Lefschetz Property (WLP) if there is an $\ell \in S_{1}$ such that for all $m$, the map $\mu_{\ell}$

$$
A_{m} \stackrel{\cdot \ell}{\longrightarrow} A_{m+1}
$$

is either injective or surjective. We assume $\operatorname{char}(\mathbb{K})=0$; as shown in [6], WLP behaves in very subtle ways in positive characteristic. In [1], Anick shows that if $r=3$ and $I$ is generated by generic forms, then $A$ has WLP. In [5], HarimaMigliore-Nagel-Watanabe introduced the syzygy bundle of $I$ to study the WLP, and this bundle also plays a key role in recent work of Brenner-Kaid [3].

Definition 1.1. If $I=\left\langle f_{1}, \ldots, f_{n}\right\rangle$ is $\left\langle x_{1}, \ldots, x_{r}\right\rangle$-primary, and $\operatorname{deg}\left(f_{i}\right)=d_{i}$, then the syzygy bundle $\mathcal{S}(I)=\widehat{\operatorname{Syz}(I)}$ is a rank $n-1$ bundle defined via

$$
0 \longrightarrow S y z(I) \longrightarrow \bigoplus_{i=1}^{n} S\left(-d_{i}\right) \stackrel{\left[f_{1}, \ldots, f_{n}\right]}{\longrightarrow} S .
$$

The cokernel of the rightmost map is $S / I$, which vanishes as a sheaf.

Definition 1.2. A vector bundle $\mathcal{E}$ on projective space is said to be semistable if for every coherent subsheaf $\mathcal{F} \subseteq \mathcal{E}$

$$
\frac{c_{1}(\mathcal{F})}{r k(\mathcal{F})} \leq \frac{c_{1}(\mathcal{E})}{r k(\mathcal{E})}, \text { where } c_{1} \text { denotes the first Chern class. }
$$

By a result of Grothendieck, every vector bundle on $\mathbb{P}^{1}$ splits as a sum of line bundles [7], so for a given line $L$, if $\mathcal{E}$ has rank $k$, then

$$
\left.\mathcal{E}\right|_{L} \simeq \bigoplus \mathcal{O}_{L}\left(a_{i}\right), \text { with } a_{1} \geq a_{2} \geq \cdots \geq a_{k} .
$$

Received by the editors July 9, 2009, and, in revised form, November 6, 2009

2010 Mathematics Subject Classification. Primary 13D02, 14J60, 13C13, 13C40, 14F05.

Key words and phrases. Weak Lefschetz property, Artinian algebra, powers of linear forms.

The first author was supported by NSF grant no. 07-07667 and NSA grant no. 904-03-1-0006.

(C)2010 American Mathematical Society Reverts to public domain 28 years from publication 
If $\mathcal{E}$ is semistable, then [7] for a generic line $L$ the tuple $\left(a_{1}, a_{2}, \ldots, a_{k}\right)$ does not vary, $\left(a_{1}, a_{2}, \ldots, a_{k}\right)$ is the generic splitting type of $\mathcal{E}$, and if $\mathcal{E}$ is semistable, then $\left|a_{i}-a_{i+1}\right| \leq 1$.

For the remainder of the paper we focus on the case $r=3$, so henceforth $S$ denotes $\mathbb{K}[x, y, z]$. In [3], Brenner and Kaid show that if $A=S / I$ is Artinian with $\mathcal{S}(I)$ semistable of generic splitting type $\left(a_{1}, \ldots, a_{n-1}\right)$, then $A$ has WLP iff $\left|a_{1}-a_{n-1}\right| \leq 1$. As a corollary of this, they recover a result of Harima-MiglioreNagel-Watanabe [5] that every Artinian complete intersection in $S$ has WLP. They also completely characterize WLP for almost complete intersections, showing that in this case if $\mathcal{S}(I)$ is not semistable, then WLP holds.

It is clear from the definition that semistability can be a difficult property to show. In this paper, we examine a special class of ideals in $S$ which falls outside the classes considered by Anick, Brenner-Kaid, and Harima-Migliore-Nagel-Watanabe. Our main result is

Theorem. An Artinian quotient of $\mathbb{K}[x, y, z]$ by powers of linear forms has WLP.

\section{Proof of the Theorem}

We begin by recalling the setup of [3. Let $\ell$ be a generic form in $S_{1}$ with $L=V(\ell)$, and let $I$ be an ideal such that $A=S / I$ is Artinian. Taking cohomology of the defining sequence for $\mathcal{S}(I)$,

$$
0 \longrightarrow \mathcal{S}(I)(m) \longrightarrow \bigoplus_{i=1}^{n} \mathcal{O}_{\mathbb{P}^{2}}\left(m-d_{i}\right) \longrightarrow \mathcal{O}_{\mathbb{P}^{2}}(m) \longrightarrow 0,
$$

we see that

$$
A=\bigoplus_{m \in \mathbb{Z}} H^{1}(\mathcal{S}(I)(m)) .
$$

On the other hand, since $\mathcal{S}(I)$ is a bundle, tensoring the sequence

$$
0 \longrightarrow \mathcal{O}_{\mathbb{P}^{2}}(m) \longrightarrow \mathcal{O}_{\mathbb{P}^{2}}(m+1) \longrightarrow \mathcal{O}_{L}(m+1) \longrightarrow 0
$$

with $\mathcal{S}(I)$ gives the exact sequence

$$
\left.0 \longrightarrow \mathcal{S}(I)(m) \longrightarrow \mathcal{S}(I)(m+1) \longrightarrow \mathcal{S}(I)\right|_{L}(m+1) \longrightarrow 0 .
$$

The long exact sequence in cohomology yields a sequence

$$
\begin{aligned}
0 \longrightarrow & H^{0}(\mathcal{S}(I)(m)) \longrightarrow H^{0}(\mathcal{S}(I)(m+1)) \stackrel{\phi_{m}}{\longrightarrow} H^{0}\left(\left.\mathcal{S}(I)\right|_{L}(m+1)\right) \\
& H^{1}(\mathcal{S}(I)(m)) \stackrel{\longleftrightarrow}{\longrightarrow} H^{1}(\mathcal{S}(I)(m+1)) \longrightarrow H^{1}\left(\left.\mathcal{S}(I)\right|_{L}(m+1)\right) \\
& H^{2}(\mathcal{S}(I)(m)) \stackrel{\longrightarrow}{\longleftrightarrow} H^{2}(\mathcal{S}(I)(m+1)) \longrightarrow H^{2}\left(\left.\mathcal{S}(I)\right|_{L}(m+1)\right)=0 .
\end{aligned}
$$

Therefore injectivity of $\mu_{\ell}$ follows from surjectivity of $\phi_{m}$, and surjectivity of $\mu_{\ell}$ from injectivity of $\psi_{m}$. Our next step is to analyze $\left.\mathcal{S}(I)\right|_{L}$. To do this, we tensor the defining sequence

$$
0 \longrightarrow S y z(I) \longrightarrow \bigoplus_{i=1}^{n} S\left(-d_{i}\right) \longrightarrow I \longrightarrow 0
$$


with $S / \ell$, yielding the sequence

$$
0 \longrightarrow \operatorname{Tor}_{1}^{S}(I, S / \ell) \longrightarrow S y z(I) \otimes S / \ell \longrightarrow \bigoplus_{i=1}^{n} S / \ell\left(-d_{i}\right) \longrightarrow I \otimes S / \ell \longrightarrow 0 .
$$

Now $\operatorname{Tor}_{1}^{S}(I, S / \ell)=0$, since it is the kernel of

$$
I \stackrel{\cdot \ell}{\longrightarrow} I(1) \text {. }
$$

After a change of coordinates, $\ell=x$ is generic. Reducing the defining equations of $I \bmod x$, we see that $S y z(I) \otimes S / \ell$ is the module of syzygies on $I \otimes S / \ell$, an ideal generated by powers of linear forms in two variables. We make use of the following pair of lemmas from [4] on ideals

$$
J=\left\langle l_{1}^{\alpha_{1}}, \ldots, l_{t}^{\alpha_{t}}\right\rangle \subseteq \mathbb{K}[y, z]=R,
$$

generated by powers of pairwise linearly independent forms.

Lemma 2.1. Let $0<\alpha_{1} \leq \alpha_{2} \cdots \leq \alpha_{t}$. Then for $m \geq 2$ :

$$
l_{m+1}^{\alpha_{m+1}} \notin\left\langle l_{1}^{\alpha_{1}}, \ldots, l_{m}^{\alpha_{m}}\right\rangle \Leftrightarrow \alpha_{m+1} \leq \frac{\sum_{i=1}^{m} \alpha_{i}-m}{m-1} .
$$

Lemma 2.2. If $J$ is minimally generated by $\left\{l_{1}^{\alpha_{1}}, \ldots, l_{t}^{\alpha_{t}}\right\}$ and $t \geq 2$, then the socle degree of $\mathbb{K}[y, z] / J$ is $\omega=\left\lfloor\frac{\sum_{i=1}^{t} \alpha_{i}-t}{t-1}\right\rfloor$, and $J$ has minimal free resolution

$$
0 \longrightarrow R(-\omega-2)^{a} \oplus R(-\omega-1)^{t-1-a} \longrightarrow \bigoplus_{i=1}^{t} R\left(-\alpha_{i}\right) \longrightarrow J \longrightarrow 0,
$$

where $a=\sum_{i=1}^{t} \alpha_{i}-(t-1)(\omega+1)$.

Proposition 2.3. If $I=\left\langle l_{1}^{d_{1}}, \ldots, l_{n}^{d_{n}}\right\rangle \subseteq S$ satisfies

$$
d_{t+1} \leq \frac{\sum_{i=1}^{t} d_{i}-t}{t-1}
$$

for all $t>1$, then $S / I$ has $W L P$.

Proof. By Lemma 2.1, the restriction $I \otimes S / \ell$ has the same number of minimal generators and degrees as $I$, and so it follows from Lemma 2.2 that

$$
\left.\mathcal{S}(I)\right|_{L} \simeq \mathcal{O}_{L}(-\omega-2)^{a} \oplus \mathcal{O}_{L}(-\omega-1)^{n-1-a},
$$

with

$$
\omega=\left\lfloor\frac{\sum_{i=1}^{n} d_{i}-n}{n-1}\right\rfloor \text { and } a=\sum_{i=1}^{n} d_{i}-(n-1)(\omega+1) .
$$

Suppose $m<\omega$. Then

$$
H^{0}\left(\left.\mathcal{S}(I)\right|_{L}(m+1)\right) \simeq H^{0}\left(\mathcal{O}_{L}(m-1-\omega)\right)^{a} \oplus H^{0}\left(\mathcal{O}_{L}(m-\omega)\right)^{n-1-a}=0,
$$

so $\mu_{\ell}$ is injective. If instead $m \geq \omega$, by Serre duality

$$
H^{1}\left(\left.\mathcal{S}(I)\right|_{L}(m+1) \simeq H^{0}\left(\mathcal{O}_{L}(-m-1+\omega)\right)^{a} \oplus H^{0}\left(\mathcal{O}_{L}(-m-2+\omega)\right)^{n-1-a}=0,\right.
$$

and thus $\mu_{\ell}$ is surjective.

Theorem 2.4. If $I=\left\langle l_{1}^{d_{1}}, \ldots, l_{n}^{d_{n}}\right\rangle \subseteq S$, then $S / I$ has $W L P$. 
Proof. If

$$
d_{t+1} \leq \frac{\sum_{i=1}^{t} d_{i}-t}{t-1},
$$

for all $t>1$, then the theorem follows from Proposition 2.3, so let $d_{1} \leq d_{2} \leq \cdots \leq d_{n}$ and suppose that $t+1$ is the first index where

$$
d_{t+1}>\frac{\sum_{i=1}^{t} d_{i}-t}{t-1} \geq\left\lfloor\frac{\sum_{i=1}^{t} d_{i}-t}{t-1}\right\rfloor=\omega .
$$

Thus, $d_{i} \geq \omega+1$ when $i \geq t+1$. If $d_{i} \in\{\omega+1, \omega+2\}$ for all $i \geq t+1$, then the shifts appearing in $\left.\mathcal{S}(I)\right|_{L}$ are as in equation (11), so the argument of Proposition 2.3 works. Suppose $k \geq t+1$ is the first index such that $d_{k} \geq \omega+3$. Then

$$
\left.\mathcal{S}(I)\right|_{L} \simeq \mathcal{O}_{L}(-\omega-1)^{a} \oplus \mathcal{O}_{L}(-\omega-2)^{b} \bigoplus_{i=k}^{n} \mathcal{O}_{L}\left(-d_{i}\right),
$$

with $a+b=k-2$. If $m<\omega$, the argument of Proposition 2.3 shows that $\mu_{\ell}$ is injective, so suppose $m \geq \omega$. We show $\psi_{m}$ is injective by a dimension computation. From the defining sequence for $\mathcal{S}(I)$ we obtain

$$
0 \longrightarrow H^{2}(\mathcal{S}(I)(m)) \longrightarrow H^{2}\left(\bigoplus_{i=1}^{n} \mathcal{O}_{\mathbb{P}^{2}}\left(m-d_{i}\right)\right) \longrightarrow H^{2}\left(\mathcal{O}_{\mathbb{P}^{2}}(m)\right) \longrightarrow 0 .
$$

By Serre duality, $h^{2}\left(\mathcal{O}_{\mathbb{P}^{2}}(m)\right)=h^{0}\left(\mathcal{O}_{\mathbb{P}^{2}}(-m-3)\right)=0$ since $m \geq \omega>0$, and

$$
h^{2}(\mathcal{S}(I)(m))=\sum_{i=1}^{n}\left(\begin{array}{c}
d_{i}-m-1 \\
2
\end{array}\right) \text { and } h^{2}(\mathcal{S}(I)(m+1))=\sum_{i=1}^{n}\left(\begin{array}{c}
d_{i}-m-2 \\
2
\end{array}\right) \text {. }
$$

Thus,

$$
\operatorname{dim} \operatorname{im}\left(\psi_{m}\right)=\sum_{i=1}^{n} \max \left(d_{i}-m-2,0\right) .
$$

The contributions come from those $d_{i} \geq m+3 \geq \omega+3$. Our assumption is that

$$
\left.\mathcal{S}(I)\right|_{L} \simeq \mathcal{O}_{L}(-\omega-1)^{a} \oplus \mathcal{O}_{L}(-\omega-2)^{b} \bigoplus_{i=k}^{n} \mathcal{O}_{L}\left(-d_{i}\right),
$$

with $a+b=k-2$. Thus for $m \geq \omega$,

$$
\begin{aligned}
h^{1}\left(\left.\mathcal{S}(I)\right|_{L}(m+1)\right) & =\sum_{i=k}^{n} h^{1}\left(\mathcal{O}_{L}\left(-d_{i}+m+1\right)\right)+h^{1}\left(\mathcal{O}_{L}(m-\omega)^{a}\right)+h^{1}\left(\mathcal{O}_{L}(m-\omega-1)^{b}\right) \\
& =\sum_{i=k}^{n} h^{1}\left(\mathcal{O}_{L}\left(-d_{i}+m+1\right)\right) \\
& =\sum_{i=k}^{n} h^{0}\left(\mathcal{O}_{L}\left(d_{i}-m-3\right)\right) \\
& =\sum_{i=k}^{n} \max \left(d_{i}-m-2,0\right) .
\end{aligned}
$$

Since this is equal to $\operatorname{dim} \operatorname{im}\left(\psi_{m}\right), \psi_{m}$ is an inclusion, so that $\mu_{\ell}$ is surjective.

It follows from Theorem 2.4 that ideals generated by powers of linear forms in $\mathbb{K}[x, y, z]$ which have unstable syzygy bundles always have WLP. As noted earlier Brenner and Kaid show that almost complete intersections with unstable syzygy bundles have WLP. Thus, it seems reasonable to ask if every ideal in $\mathbb{K}[x, y, z]$ with unstable syzygy bundle has WLP. 
Example 2.5. For the ideal $I=\left\langle x^{5}, y^{5}, z^{5}, x^{2} y z, x y^{2} z\right\rangle \subseteq \mathbb{K}[x, y, z], \mathcal{S}(I)$ is not semistable, by Proposition 2.2 of [2]. The Hilbert function of $A$ is $(1,3,6,10,13,13$, $10,6,3)$, and a computation shows the map from $A_{4} \rightarrow A_{5}$ is not full rank, so $A$ does not have WLP.

As noted, Theorem 2.4 need not hold for more than three variables:

Example 2.6. The $\operatorname{ring} A=\mathbb{K}[x, y, z, w] /\left\langle x^{3}, y^{3}, z^{3}, w^{3},(x+y+z+w)^{3}\right\rangle$ appears in Example 8.1 of [ $[6$ and does not have WLP. The Hilbert function of $A$ is $(1,4,10,15,15,6)$, and a computation shows the map from $A_{3} \rightarrow A_{4}$ is not full rank. So WLP need not hold for powers of linear forms in more than three variables.

\section{CONCLUDing REMARKS}

The proof of Theorem 2.4 works for any ideal which has the same splitting type as an ideal generated by powers of linear forms, so it would be interesting to find families of such ideals. In light of Example 2.6. we ask: are there reasonable additional hypotheses so that a version of Theorem 2.4 does hold in more than three variables? A second question is if ideals generated by powers of linear forms possess the Strong Lefschetz Property. As pointed out by the referee, the answer is no: SLP fails for the ideal generated by cubes of four general linear forms and for multiplication by a cube of a linear form. However, multiplication by a general form of degree three does have maximal rank, so we ask: does multiplication by a general form of any degree induce a multiplication having maximal rank?

\section{ACKNOWLEDGEMENTS}

Computations were performed using Macaulay2, by Grayson and Stillman, available at: http://www.math.uiuc.edu/Macaulay2/. Scripts to analyze WLP are available at: http://www.math.uiuc.edu/ asecele2. We thank an anonymous referee for thoughtful comments.

\section{REFERENCES}

[1] D. Anick, Thin algebras of embedding dimension three, J. Algebra, 100 (1986), 235-259. MR839581 (88d:13016a)

[2] H. Brenner, Looking out for stable syzygy bundles, Adv. Math., 219 (2008), 401-427. MR2435644 (2009g:14049)

[3] H. Brenner, A. Kaid, Syzygy bundles on $\mathbb{P}^{2}$ and the weak Lefschetz property, Illinois J. Math., 51 (2007), 1299-1308. MR2417428 (2009j:13012)

[4] A. Geramita, H. Schenck, Fat points, inverse systems, and piecewise polynomial functions, J. Algebra, 204 (1998), 116-128. MR1623949 (99d:13019)

[5] T. Harima, J. Migliore, U. Nagel, J. Watanabe, The weak and strong Lefschetz properties for Artinian $\mathbb{K}$-algebras, J. Algebra, 262 (2003), 99-126. MR1970804 (2004b:13001)

[6] J. Migliore, R. Miró-Roig, U. Nagel, Monomial ideals, almost complete intersections and the weak Lefschetz property, Trans. Amer. Math. Soc., to appear.

[7] C. Okonek, M. Schneider, H. Spindler, Vector Bundles on Complex Projective Spaces, Progress in Mathematics, vol. 3, Birkhäuser, Boston, 1980. MR561910 (81b:14001)

Department of Mathematics, University of Illinois, Urbana, Illinois 61801

E-mail address: schenck@math.uiuc.edu

Department of Mathematics, University of Illinois, Urbana, Illinois 61801

E-mail address: asecele2@math.uiuc.edu 